\title{
Organismo y acción situada: mapeando el concepto de affordance*
}

\author{
Organism and Situated Action: Mapping the Concept of Affordance \\ Organismo e ação situada: mapeando o conceito de affordance
}

\author{
Pablo López-Silva** \\ Universidad de Valparaíso
}

Doi: https://doi.org/10.12804/revistas.urosario.edu.co/apl/a.7475

\section{Resumen}

En el contexto de la comprensión de la interacción organismo-medio ambiente, el concepto de affordance designa las diversas oportunidades para la acción a las que la percepción de un objeto invita. Cuando miramos un muro, no solo percibimos sus propiedades físicas brutas (color, tamaño, localización, etc.), sino también ciertas cualidades, como que es escalable, dependiendo del contexto en que tal percepción ocurra. Dicha noción de escalabilidad integrada en mi percepción del muro es una affordance. Durante los últimos años, este concepto se ha vuelto popular en diversas disciplinas; sin embargo, no ha estado acompañado de un desarrollo unificado y preciso de su comprensión. Este artículo formula y examina algunos de los debates más fundamentales el torno al concepto de affordance. Luego de evaluar críticamente algunas de las ideas más paradigmáticas dentro de estas discusiones, se proponen algunos desafíos que enfrenta el desarrollo del término para, finalmente, explorar algunas formas en que se pueden afrontar tales desafíos.

Palabras clave: percepción, affordances, psicología ecológica, acción. fbstract

In the context of the understanding of environment-organism interactions, the concept of affordance refers to the different opportunities for action that the perception of an object invites. When we see a wall, we do not only perceive its brute physical properties (such as color, size, location, etc.), but we perceive it as climbable, depending on the context in which such perception occurs. The notion of climbability embedded into the perception of the wall is known as affordance. Over the last years, this concept has become popular within a number of disciplines; however, this has not been accompanied by a precise and unified conceptual development of the term. This article formulates and examines some of the most fundamental debates surrounding the understanding of the current concept of affordance. After critically evaluating some of the main tenets within these discussions, some of the challenges posed by the new concept are discussed, as well as ways in which htye can be faced.

Keywords: Perception, affordances, ecological psychology, action.

* La escritura final de este trabajo se realizó en el marco del Proyecto FONDECYT No 111605442 "La arquitectura agencial del pensamiento humano", otorgado por la Comisión Nacional de Investigación Científica y Tecnológica (Conicyt) del Gobierno de Chile. El autor agradece a Tom McClleland, Jöelle Proust, Manuel Heras-Escribano y los revisores ciegos provistos por la revista, por sus comentarios provistos en versiones preliminares de este trabajo.

** Dirigir la correspondencia a Pablo López-Silva, Escuela de Psicología, Universidad de Valparaíso, Chile. Correo electrónico: lopez.silva@gmail.com 


\section{Resumo}

No contexto da compreensão da interação organismo-meio ambiente, o conceito de affordance designa as diversas oportunidades para a ação que a percepção de um objeto convida. Quando olhamos um muro, não só percebemos suas propriedades físicas brutas (cor, tamanho, localização, etc.), mas também podemos percebê-lo como escalável, dependendo do contexto em que essa percepção ocorre. À noção de escalabilidade integrada em minha percepção do muro denomina-se lhe affordance. Durante os últimos anos, este conceito se tem tornado popular em diversas disciplinas, no entanto, isto não tem estado acompanhado de um desenvolvimento unificado e preciso de sua compreensão. Este artigo formula e examina alguns dos debates mais fundamentais que rodeiam o entendimento atual do conceito de affordance. Após avaliar criticamente algumas das ideias mais paradigmáticas dentro destas discussões, propõem-se alguns desafios que enfrenta o desenvolvimento do termo para finalmente explorar algumas formas em que tais desafios poder ser afrontados.

Palavras-chave: percepção, affordances, psicologia ecológica, ação.

En el contexto de los estudios de la percepción humana desde una perspectiva ecológica, Gibson (1979) introduce originalmente el concepto de affordance para designar las diversas oportunidades de acción que la percepción de un objeto en el ambiente invita. La idea central tras esta propuesta es que la percepción humana no solamente despliega las propiedades físicas brutas de sus objetos intencionales (forma, tamaño, color, localización, etc.), sino que también incorpora la percepción de potenciales acciones. Por ejemplo, cuando estoy frente a un árbol, mi percepción visual no solamente me informa de las propiedades físicas del árbol tales como su altura, colores, textura, posición, etc., sino que también - dependiendo del contexto de la interacción y de mis propias capacidades físicas - uno podría percibir el árbol como "escalable". A la propiedad de "escalabilidad" contenida en mi percepción del árbol, Gibson (1979) la denominará affordance. Durante los últimos años, el uso de este concepto ha trascendido las fronteras de la psicología y ha influido en el desarrollo de diversas disciplinas como las neurociencias (Cisek \& Kalaska, 2010); la teoría musical (Krueger, 2011); la antropología (Inglod, 2011); la teoría del diseño (Norman, 1999); la inteligencia artificial (Horton, Chakraborty, \& Amant, 2012); la fenomenología (Dreyfus, 2002) y la filosofía de la mente (Siegel, 2010; 2014), entre muchas otras. No obstante, esta popularidad en el uso del término no ha estado acompañada de un respectivo desarrollo conceptual unificado y preciso del mismo. Por ejemplo, específicamente en español, el término affordance ha intentado ser traducido como "ofrecimiento", "disponibilidades" o "facilitaciones". Sin embargo, además de poseer un nulo impacto en la literatura, tales traducciones no expresan algunas de las dimensiones más fundamentales del concepto original. Por esto, en este artículo referiremos al término en su forma original y desde esta discutiremos los debates que rodean su entendimiento. Así, a la luz de este y otros problemas Este artículo formula y examina algunos de los debates más fundamentales que rodean el entendimiento actual del concepto de affordance. Luego de evaluar críticamente algunas de las ideas más paradigmáticas dentro de estas discusiones para así mapear el desarrollo del concepto, se proponen algunos desafíos que nacen de tales discrepancias y nalmente se exploran algunas potenciales aplicaciones a nuevas áreas de investigación.

\section{El problema preliminar: la multiplicidad tipológica de las affordances}

Uno de los problemas preliminares con los que uno se encuentra al introducirse al concepto tiene que ver con la proliferación de múltiples 
tipologías de affordances en la literatura. Existen muchas voces diciendo cosas distintas respecto de lo que el término implica. Tal vez, en sí misma tal multiplicidad no sea un problema, ya que podría capturar la complejidad del concepto; sin embargo, muchas veces la relación entre tales tipologías propone un desafío comprensivo por ser muy obscura. Finalmente, esta multiplicidad inevitablemente genera problemas, ya que no es claro si aquello a lo cual tales tipologías refieren siquiera podría ser denominado como affordances. Por ejemplo, Proust (2016) refiere al concepto affordance-sensing como una evaluación no consciente de la posibilidad de realizar una tarea propuesta por el ambiente de forma exitosa. Este tipo de affordance buscaría predecir beneficios o riesgos en base a señales percibidas desde el ambiente contrastadas con las capacidades cognitivas y físicas del sujeto. Para dicho autor, las affordance-sensings pueden ser impulsivas (desencadenada por sentimientos) o habituales (desencadenada por oportunidades detectadas de forma innata o aprendida). En ambos casos, una affordance-sensing está orientada a una acción inmediata y no generaría inferencias respecto a las consecuencias de las potenciales acciones a largo plazo. Para Proust (2016), las affordance-sensings proporcionan una estructura de representación adecuada para que una señal se produzca con fluidez y se entienda como un disparador de acción, de esta forma, complementando la noción gibsoniana que revisaremos en la siguiente sección, las affordance-sensings serían más primitivas que las representaciones puramente descriptivas, serían una condición para estas (p. 185).

Dentro de esta formulación de la noción de affordance, Proust (2016) propone tres categorías: (1) affordances sociales (social affordances), entendidas como aquellas que sirven para que los infantes logren compartir emociones con sus cuidadores; (2) affordances comunicacionales (communication affordances), que servirían para la elección eficiente de los términos precisos para transmitir información, y (3) affordances epistémicas (epistemic affordan- ces), que servirían para recibir información desde el ambiente. Esta última también es llamada affordance cognitiva y podría ser de vital importancia en el proceso del desarrollo de un protolenguaje. Sea cual sea el caso, la visión de Proust en su definición del concepto parece ser evaluativa i.e., las affordances son evaluaciones inconscientes de la autoeficacia de un organismo respecto de una tarea que el ambiente demanda.

Por su parte, Scarantino (2003) propone otra taxonomía para categorizarlas. Las affordances dirigidas a objetivos (goal-affordances) son aquellas que se constituyen como una acción (doing), y las affordance eventuales (happening affordance) se presentan simplemente como otras posibilidades de acción cuando ya se está realizando una acción fundamental. Scarantino (2003) también distingue entre affordances infalibles (que ocurren el 100\% de las veces que aparecen en la percepción de un sujeto) y affordance probabilísticas, las cuáles poseen menos probabilidades de ser actualizadas en la acción esperada. Así mismo, para esta autora, el valor del concepto no surge de su capacidad de evaluar la autoeficacia, es más, las affordances no juegan tal rol, sino que guían la acción a partir de la percepción consciente de oportunidades en el ambiente. Si bien Scarantino pone el énfasis en cómo una affordance es constitutiva de una acción, este enfoque parece ser complementario con el de Proust, quien lograría explicar cómo ocurriría esto.

Ahora bien, Rietveld y Kiverstein (2014) se refieren al concepto de affordances socioculturales, definidas como potenciales acciones que necesariamente incluyen componentes exploratorios, mediacionales y cooperativos alojados en un contexto cultural. La idea es que la cultura provee el espacio y el lugar para que la percepción de ciertas oportunidades en el ambiente sea siquiera inteligible. Finalmente, Gendler (2008) propone reemplazar el término affordance por el de Alief, entendido como un estado cognitivo caracterizado por la generación de una propensión innata o habitual para responder a un estímulo, teniendo en 
cuenta una alternativa para comprender las reacciones que se rigen por mecanismos automáticos. Si bien la propuesta ha sido explorada durante los últimos años, es claro que esta parece alejarse del concepto original de affordance.

Tal como podemos observar hasta este punto, el principal desafío en este contexto implica clarificar la relación entre las diferentes taxonomías antes mencionadas. Para esto, es necesario dilucidar si todas realmente refieren al mismo aspecto de la realidad o si en el proceso de construir tales taxonomías: (1) se construyen visiones dispares del concepto o terminan (2) construyéndose nuevos conceptos. Prima facie, las taxonomías ofrecidas por Proust, Scarantino y Rietveld y Kiverstein no parecen del todo dispares, sino que apuntan a diferentes dimensiones del mismo concepto. Ahora bien, este desafío requiere una mayor formulación de todas las ideas de los autores y definir los puntos de convergencia. Para poder clarificar este asunto, en la siguiente sección revisaré algunos de los componentes que parecen ser fundamentales para que un concepto clasifique como affordance a partir de las ideas originales de Gibson (1966; 1979). Ese análisis podría ayudar a evaluar la plausibilidad de las taxonomías descritas en esta sección.

\section{El problema terminológico-descriptivo}

El concepto de affordance se introduce en la tradición de la psicología ecológica de Gibson (1966; 1979), la cual centrará su estudio en la interacción entre un organismo y su medio ambiente, y en cómo tal intercambio propicia y posibilita ciertas dinámicas estructurales, conductuales y psicológicas en interdependencia y cambio constante (Turvey, 1992). Esta idea tendrá al menos dos consecuencias a nivel ontológico. Primero, el sujeto de la psicología no puede ser entendido fuera de su ambiente, y el concepto de "ambiente" tampoco puede ser entendido sin tomar en cuenta las capacidades de los agentes que lo habitan para modificarlo. Ambas nociones son parte de una relación simbiótica en la cual todo proceso mental es inteligible. En consecuencia, toda actividad humana debe ser entendida en el contexto de la interacción en la cual ocurre. Segundo, y más importante, el cambio propuesto por la psicología ecológica de Gibson promueve la existencia de entidades que emergen solamente en la interacción entre organismo y ambiente, y que, por lo tanto, serían entidades necesariamente como interaccionales; el mejor ejemplo de este tipo de entidades son las affordances. A esta idea le llamaré el "principio interaccional de las affordances". Para entender este principio, es necesario tener en cuenta que Gibson (1979) indica que la emergencia de las affordances: "implica la necesaria complementariedad entre el animal y su medio ambiente" (p. 119), ya que tanto sujeto - como poseedor de habilidades que hacen posibles ciertas acciones-, como ambiente - en tanto posibilitador de la emergencia de ciertas potenciales acciones en virtud de sus características objetivas - son condiciones igualmente necesarias para el despliegue de las affordances en el campo perceptual de un organismo. Así, las affordances no son propias del ambiente ni de la subjetividad de los agentes, sino que emergen de la dinámica ambiente-individuo. Es importante señalar que, desde el punto de vista de la filosofía de la ciencia, la introducción del concepto original de affordance propone una distinción losó ca fundamental para la demarcación de la psicología como ciencia que ofrece un nivel explicativo irreductible al promover la existencia de entidades que solamente serían inteligibles en la relación entre el sujeto y su medio ambiente, dominio al cual, por lo demás, la psicología tendría un acceso privilegiado.

Pues bien, el debate más fundamental que rodea al concepto de affordance es justamente el problema de su definición y el problema de la descripción de las propiedades básicas que nutren tal definición. Llamaré a este, el "problema 
terminológico-descriptivo", ya que implica la definición del término mediante la descripción de sus propiedades más fundamentales. Si bien Gibson $(1966,1979)$ provee la descripción original del concepto de affordance, es claro que su trabajo deja espacio para muchas interpretaciones, algunas de ellas incluso contradictorias (Heft, 2001; Michaels, 2003; Scarantino, 2003; Withagen, Poel, Araujo \& Pepping, 2012). Una potencial estrategia para zanjar varios de los debates que rodean el concepto de affordance podría implicar una exégesis del trabajo original de Gibson. Sin embargo, según algunos, la noción inicial de affordance fue tan poco desarrollada que tal estrategia podría empobrecer la discusión limitando diferentes comprensiones provenientes de otras disciplinas que podrían nutrir positivamente el debate (Jenkins, 2008). En mi opinión, acá estamos frente a una decisión histórico-metodológica. El concepto fue introducido por Gibson dentro de un enfoque específico, y a lo largo de toda su obra se puede comprender el contexto filosófico que lo sustenta. Por lo tanto, creo que al momento de discutir las propiedades fundamentales del concepto uno debe respetar su "denominación de origen" y encausar la discusión en torno a lo que aparece o no en la obra de Gibson. Esto, potencialmente, podría llevarnos a una clarificación del concepto que respete las intenciones iniciales de Gibson y permita generar una diferenciación en medio del océano de definiciones observadas en la sección anterior.

En términos generales, las affordances son descritas en la literatura como "oportunidades para la acción". Sin embargo, tal definición carece de la especificidad necesaria como para, entre otras cosas, guiar un programa de investigación experimental en psicología lo suficientemente demarcado. Por esto, McGrenere y Ho (2000) indican que es posible establecer al menos tres características principales de las affordances siguiendo la formulación gibsoniana original:
1. Las affordances existirían en relación con las capacidades de un agente en particular.

2. La existencia de una affordance sería independiente a la capacidad del sujeto de percibirlo.

3. Una affordance no cambiaría como lo hacen las necesidades y objetivos del actor.

Respecto de (1), claramente la percepción de la "pateabilidad" de un balón se verá condicionada por las capacidades que el agente posea, por ejemplo, tener sus pies en condiciones que permitan realizar la potencial acción a la que el balón invita. El mismo muro que para mí puede ser escalable, podría no serlo para un niño, esto depende de las diferentes capacidades físicas de cada uno. Una de las principales características de una affordance es que su presencia en el campo perceptual de un individuo está supeditada a las habilidades físicas que permitirían a este sujeto realizar la acción invitada por el objeto, incluso si esta no se realiza. De esta manera, el concepto de affordance es clave para explicar la fluidez del comportamiento humano-animal no deductivo en sus respectivos ambientes.

Complementando esta idea, Whithagen et al. (2012) indican que las affordances no solo implicarían la percepción de potenciales acciones, sino la invitación a actuar de cierta forma. La idea es que una affordance también está compuesta por una "solicitud" (solicit) ya que los agentes se sienten inmediatamente atraídos a actuar de cierta forma $-\mathrm{y}$ no de otras también posiblesante ciertos estímulos dependiendo del contexto en el cual surge tal necesidad (Dreyfus \& Kelly, 2007). Aunque no está exento de críticas, este aspecto podría explicar no solo la fluidez de la acción humana, sino su especificidad por medio del establecimiento de un sistema jerárquico de potenciales acciones en virtud de su eficiencia adaptativa-contextual. Así, un sujeto se sentiría más inclinado a actuar de cierta forma, no solo por sus necesidades psicológicas, sino por el tipo de desafío que el ambiente le impone, premisa que 
parece consistente con el paradigma ecológico y la propuesta gibsoniana original.

Ahora bien, desde el punto de vista gibsoniano, la premisa (2) parece problemática. Simplemente, parece un error indicar que la existencia de una affordance es independiente de la capacidad del sujeto de percibirla, ya que, por definición, una affordance se constituye como tal en tanto percepción de una oportunidad para la acción (Gibson, 1966). La presencia de una affordance en el campo perceptual de un sujeto necesariamente depende de que este pueda percibirla, ya que sin percepción de una affordance, no hay presencia de tal affordance en el campo perceptual del sujeto. De esta forma, la premisa 2) debería ser rechazada simplemente por ser contradictoria, siguiendo la formulación gibsoniana original. Así como la capacidad de un organismo $\mathrm{O}$ de ver un objeto $\mathrm{Y}$ teniendo ciertas propiedades de tipo $\mathrm{P}$ depende de la capacidad perceptual de $\mathrm{O}$, si las affordances son acciones percibidas en el ambiente, su presencia necesariamente depende de las capacidades de $\mathrm{O}$ de percibir el ambiente de cierta forma.

Tal como señala McClelland (2017a), la idea de que algunos objetos en el ambiente presentan oportunidades para la acción, y que agentes sean sensibles perceptualmente a tales oportunidades, debería ser bastante inocua. Es más, es difícil ver como un agente podría actuar eficientemente en su ambiente sin percibir tales oportunidades para la acción. Toda la idea del concepto de affordance refiere a la percepción directa de oportunidades para la acción en el medio ambiente. Tal como indican Withagen et al. (2012), una affordance puede ser una invitación a actuar solo si un agente la percibe. Ahora, si las affordances no son percibidas, ellas no tendrían el potencial de atraer la acción adecuada por parte del agente. Cuando observamos un árbol, nosotros no somos conscientes de sus propiedades brutas y luego "deducimos" que podría ser escalable (salvo excepciones). Simplemente, el árbol es percibido como escalable, y este hecho logra explicar la fluidez de nuestro comportamiento en el ambiente. Por otra parte, el indicar que la existencia de una affordance sería independiente a la capacidad del sujeto de percibirlo contraviene el principio interaccional, ya que las affordances surgirían como una propiedad adosada a un objeto específico de la percepción, por lo que no hay razones suficientes para apoyar la premisa (2) sin alejarse de forma relevante de la definición propuesta por Gibson.

Ahora bien, la premisa (2) podría ser reformulada. Pensemos que la presencia de una affordance en el campo perceptual de un sujeto es independiente del desarrollo conceptual, narrativo, interpretativo y atencional de este (los que estarían a la base de potenciales interpretaciones y explicaciones sobre las propiedades de los objetos de la percepción). No es controversial indicar que un bebé, sin todas estas capacidades completamente desarrolladas, puede percibir un sillón como "escalable", un vaso como "tomable" o un balón como "pateable". Al menos desde un punto de vista conductual, es plausible interpretar el comportamiento de un niño, incluyendo tales elementos en su percepción del mundo, sin tener los conceptos respectivos. Es más, esto parece ser la forma más parsimoniosa para explicar el comportamiento exploratorio de los infantes. Sin embargo, en cada caso, la presencia de tales affordances dependerá de las capacidades físicas del bebé, y esta reformulación parece consistente con la idea de que estas son percibidas directamente -i.e., sin mediación de representaciones (Horton et al. 2012) — y que nuestra percepción de tales oportunidades para la acción sería integral para explicar "el cómo y el porqué actuamos de la manera que lo hacemos" (McClelland, 2017b, p. 3).

Vera y Simon (1993) parecen oponerse a esta idea indicando que esta visión ingenua de la percepción es el producto final de complejos mecanismos que buscan codificar patrones de estímulos en el entorno de un organismo. No obstante, esto no parece ser nada más que un falso dilema. Uno puede decir sin riesgo a contradicción que la codificación de los 
patrones de estímulos perceptuales que un aparato cognitivo desarrolla a lo largo de su desarrollo ontogenético se manifiesta fenoménicamente en la percepción como affordances, por lo que la idea de estos autores podría ser integrada a la noción de affordance sin problemas. La crítica de Simon y Vera (1993) nace de la negación del principio interaccional, para estos autores, las affordances son representacionales funcionales internas que resultan de este proceso de codi cación de los estímulos perceptuales, y por lo tanto, estas están en la 'cabeza' del sujeto. El problema con esto es que tal negación trivializa completamente la noción de affordance, que pierde cualquier relación con su formulación original. Pues bien, mi objetivo acá no es salvar esta segunda idea a toda costa, pero es claro que puede ser reinterpretada en formas que parecen ser operativas desde el punto de vista gibsoniano.

¿Qué ocurre con la idea de que una affordance no cambiaría como lo hacen las necesidades y objetivos del actor i.e., premisa (3)? Esta idea parece estar claramente presente en la formulación original de Gibson (1982):

The affordances of the environment are permanent, although they do refer to animals and are species-specific. The positive and negative valences of things that change when the internal state of the observer changes are temporary. The perception of what something affords should not be confused with the "coloring" of experience by needs and motives. Tastes and preferences fluctuate. Something that looks good today may look bad tomorrow but what it actually offers the observer will be the same. (p. 410)

Gibson (1966) es claro en distinguir el término affordance del término "valencia", este último entendido como la valoración (positiva o negativa) de una situación u objeto en el mundo. El autor indica que: "he acuñado esta palabra [affordance] como sustituta de valores [valence], un término que conlleva una vieja carga de significado filosófico. Quiero decir simplemente lo que las cosas proveen, para lo bueno o lo malo. Lo que permite al observador, después de todo, depende de sus propiedades" (p. 89).

Ahora bien, el hecho de que esta idea esté contenida en la formulación original del término no la hace inmune a críticas. Es cierto que yo puedo identificar un muro como "escalable", independiente de si estoy triste o alegre. Es cierto que la valencia de un objeto o situación parece ser separable de las oportunidades de acción que tales objetos o situaciones invitan. Mas, existen buenas razones para pensar que la naturaleza de una situación u objeto perceptual junto con el contexto de cierta tarea adaptativa podrían influir y hasta determinar la presencia de ciertas affordances en el campo perceptual de un sujeto por sobre otras.

La investigación exploratoria en psicopatología fenomenológica ha mostrado que sujetos en estados prepsicóticos y psicóticos con problemas de procesamiento afectivo experimentan transformaciones perceptuales en la forma en que el mundo y sus objetos se presentan a la conciencia. Esto ha sido interpretado como una variación en las affordances presentes en el campo perceptuales de tales sujetos (Fuchs, 2005). Kusters (2014) indica que la percepción del cuerpo y los pensamientos en las etapas previas a la aparición de delirios psicóticos invita a acciones nunca antes percibidas. Un ejemplo claro de esto ocurre en las atribuciones de agencia. En situaciones no-patológicas, nuestros movimientos corporales son comúnmente acompañados por sensaciones de control, fluidez y agencia, entre muchas otras (Bayne, 2008; 2011). Es más, en tales situaciones, la pregunta sobre quién es el autor de nuestros movimientos corporales no es siquiera un asunto. Sin embargo, pacientes en riesgo de formar delirios de control externo - entre otras alteraciones de la agencia motora-, indican que, previo a la aparición del delirio propiamente tal, la sensación de su cuerpo y de toda su realidad material invita a acciones nuevas (Fuchs, 2005; Payne, 2013). En tales momentos, por ejemplo, la pregunta sobre quién 
tiene el control de sus movimientos corporales aparece como un asunto relevante y conflictivo en la vida mental de los pacientes (Conrad, 1958). Por lo que la "agenciabilidad" de los movimientos corporales podría, hipotéticamente hablando, ser entendida como una affordance que surge de la necesidad de darle sentido a las acciones corporales en un ambiente fenomenológicamente enrarecido. A partir de esto, prima facie, podríamos indicar que la presencia de una affordance cambia tal como lo hacen las necesidades físicas y psicológicas y los objetivos del sujeto. Ahora, si bien este no es el caso normal, la patología acá cumple el rol de informarnos respecto de las características fundamentales de una affordance. Por ejemplo, Kusters (2014) indica que, en etapas pre-psicóticas, los propios pensamientos invitan agarrabilidad, como si se tratasen de elementos físicos en el ambiente; Fuchs (2005) indica que ciertos objetos en el ambiente, como una campana, invitan a acciones que antes no invitaban. Si bien las affordances del ambiente no parecen cambiar por nuestras apreciaciones personales de la realidad, parecen cambiar bajo ciertas situaciones límites como en las etapas pre-psicóticas, las cuáles han sido asociadas a alteraciones en el procesamiento perceptual y afectivo de diversos aspectos de la realidad (Ratcliffe, 2013). Aunque este punto es exploratorio, parece alimentar la idea de que la variabilidad de las affordances en la percepción del mundo es una posibilidad no controversial dentro del enfoque gibsoniano.

Pensemos ahora en un ejemplo menos exploratorio: por lo general, cuando estoy escribiendo, la taza de té a mi lado no se presenta así misma como "lanzable", incluso aunque yo sepa que podría lanzársela al colega ruidoso de la oficina de al lado. La mayoría de las veces, la taza se presenta como "agarrable", dado el contexto del uso que le estoy dando y mis capacidades físicas respecto de la taza. Sin embargo, si alguien entra a mi oficina y trata de atacarme, la misma taza podría presentarse como "lanzable" dada mi necesidad de defenderme de tal ataque. Ejemplos como este podrían hacernos concluir que la presencia de una affordance en el campo perceptual de un sujeto definitivamente podría variar dependiendo de las necesidades y objetivos emergentes como tareas desde el medio ambiente (Walsh, 2015).

Ahora bien, además de contravenir el principio interaccional de las affordances, la premisa (3) de McGrenere y Ho (2000) no parece explicar la variabilidad que todos experimentamos en la presencia de affordances en nuestra vida perceptual diaria. Así, es posible indicar que las affordances que la percepción despliega pueden cambiar a partir de la forma en que un agente enfrenta ciertas situaciones/objetos desde el punto de vista físico y psicológico y que, por lo tanto, estas cambian a partir de las necesidades y objetivos del agente.

Aparentemente, la naturaleza problemática de las premisas (2) y (3) de McGrenere y Ho (2000) se deriva de una confusión entre dos ideas respecto de la percepción. Para poder entender de forma correcta las propiedades y evolución de las affordances uno debe mantener clara la distinción entre cómo un cierto objeto puede presentarse en mi campo de la conciencia y en el del conocimiento que yo tengo sobre tal objeto. Las affordances pertenecerían al primer dominio i.e., la apariencia que tiene el ambiente para un agente. Ahora bien, ambos campos son distintos, y muchas veces pueden orientarse en direcciones opuestas. Muchos errores conceptuales respecto de la definición de las características de las affordances parecen tener su base en la falta de separación entre ambas dimensiones, lo que no nos permite ver que ellas no pertenecen al dominio de aquello que sabemos sobre el mundo, sino al dominio respecto de cómo el ambiente aparece ante nosotros. Si bien las affordances nos pueden ayudar a actuar efectivamente en el mundo, también nos pueden llevar a errores cuando presentan objetos invitando a acciones que objetivamente no se pueden realizar exitosamente. Tal como señala Gaver (1991), un árbol puede ser 
objetivamente escalable por mí, incluso si yo no lo percibo como escalable, o un árbol que no es realmente escalable por mí, puede aparecer como escalable. ${ }^{1}$ En ambos casos, la conclusión es que las affordances pertenecen al dominio de la apariencia que el ambiente tiene para un agente con relación a ciertas propiedades del objeto y sus propias capacidades. $^{2}$

\section{El problema del rol: affordances en fenomenología y neurociencias}

Una de las condiciones que ha nutrido los desacuerdos terminológico-descriptivos es el hecho de que, durante los últimos años, el concepto de affordance ha intentado ser definido desde al menos dos enfoques: el fenomenológico y el neurobiológico. Esta diferencia en puntos de referencia en términos explicativos parece haber generado algunos desacuerdos sobre el rol del término en la forma en que exploramos la mente humana.

La psicopatología fenomenológica provee una variedad de casos en los cuáles la apariencia del mundo difiere completamente del conocimiento objetivo que los sujetos poseen de este. Por ejemplo, pacientes psicóticos pueden llegar a informar desde el punto de vista experiencial que ciertas partes de sus cuerpos están en control de agentes externos (delirios de control), y a su vez, indicar que, si bien ellos saben que esto es imposible, es la forma en que experimentan ciertos miembros corporales (ver De Hann \& De Bruin, 2010, p. 385). Estos casos apoyan la idea de que la dimensión de las apariencias del ambiente - al cual pertenecen las affordances-, es claramente distinta de la dimensión sobre el conocimiento que se puede tener del ambiente.

2 Alguien podría indicar que las affordances ejemplifican casos de penetración cognitiva i.e., la forma en que estados disposicionales podrían contaminar el contenido fenoménico de una experiencia (Siegel, 2014). Pues, bien, incluso si este fuese el caso, el proceso de penetración cognitiva explicaría la forma en que las affordances figurarían en el campo perceptual de un sujeto, lo que es completamente consistente con la idea de que estas pertenecen al dominio de la apariencia que el ambiente tiene para un sujeto. Para una comprensión del concepto de penetración cognitiva, ver Siegel (2010).
Heft (2001) indica que el principal rol del concepto de affordance radica en que es capaz de describir de forma más precisa nuestra fenomenología perceptual. La presencia de affordances en nuestra experiencia perceptual captura la riqueza de la forma en que experimentamos el mundo y sus objetos, y la forma en que actuamos sobre este sin tener la experiencia de decisión o esfuerzo asociada a nuestras acciones más comunes en el diario vivir. Así, el concepto de affordance sería el mejor candidato para explicar aquello que quisiera llamar "fluidez conductual" respecto de las acciones corporales. Tal como indica McClelland (2017b):

The concept of affordances allows us to capture these vivid aspects of our perceptual experience. It also helps capture what it's like to act on these affordances. When we act on a soliciting affordance, we often don't explicitly decide to perform the solicited action. Our experience is not one of choosing to perform a certain action, but more of allowing one's actions to be led by the affordances perceived. One doesn't have to decide to scratch the itch, for example. If anything, one would have to decide not to and deliberately suppress the urge to scratch. (p. 5)

A pesar de que es cierto que la idea de affordance se torna descriptivamente atractiva al observar su rol en la forma de precisar nuestra fenomenología agencial, muchos discuten no solo este rol, sino su mera existencia. Desde un punto de vista conservador sobre los contenidos de la experiencia perceptual, Prinz (2012) ha sugerido que no es necesario recurrir a la existencia de affordances para explicar la fluidez conductual de un sujeto en su medio ambiente; al tener una experiencia visual de una taza, aquello que llamaríamos affordance de "agarrabilidad" (grippability) puede ser reducido a ciertos movimientos corporales anticipatorios que surgen del acto de imaginarse a uno mismo tomando la taza. Es decir, Prinz (2012) reduce la fluidez conductual al rol anticipatorio que tendría la imaginación en el cuerpo. 
Si bien algunos grados de escepticismo son sanos dentro de discusiones sobre conceptos fenomenológicamente debatibles, la crítica de Prinz no parece del todo persuasiva. El acto imaginativo, por lo general, está caracterizado por sensaciones de control, voluntariedad e intencionalidad, entre otras. Salvo en el caso del "vagabundeo mental" (mental wandering), tales sensaciones parecen ser fundamentales al acto de imaginar. Pues bien, esto no parece ser consistente con la fluidez agencial que presentan los sujetos al actuar efectivamente sobre su medio ambiente en su diario vivir. Las características fenomenológicas del acto de imaginar no parecen ser consistentes con la experiencia de actuar efectivamente en el mundo en nuestro diario vivir; cuando esto ocurre, no tenemos la experiencia de estar haciendo un esfuerzo extra o estar dirigiendo grados de concentración extra a nuestras actividades cotidianas. Por ejemplo, el acto de manipular una manilla para abrir una puerta no parece estar acompañado de sensaciones parecidas a las que acompañan el acto de imaginar y proyectar, podríamos decir que tal acto es acompañado por una sensación de automatismo. ${ }^{3}$ En cambio, la presencia de affordances en nuestra experiencia perceptual parece capturar de mejor manera la forma en que actuamos sobre el medio sin tener la experiencia de decisión o esfuerzo que la noción de Prinz requiere, y así, el concepto de affordance sigue siendo el mejor candidato para explicar la fluidez conductual de las acciones en el mundo.

Por otra parte, la propuesta de Prinz parece implicar la idea de que un sujeto que actúa efectivamente en su ambiente, lo hace solo porque "imagina" y "proyecta" sus potenciales acciones. El asunto es que los humanos actuamos constantemente de forma efectiva en el mundo, lo que implicaría estar imaginando nuestras posibilidades de acción en cada momento para poder actuar. Claramente, este

Es importante señalar que esto no implica la idea de que tal acto fenomenológica automático no sea ontológica una acción voluntaria. procesamiento imaginativo paralelo es inconsistente con nuestra fenomenología conductual-agencial, pero por sobre todo hace de la respuesta de Prinz implausible respecto de los recursos cognitivos requeridos para que cada organismo actúe efectivamente en el mundo. En otras palabras, la sugerencia de Prinz no parece ser cognitivamente económica. Tal vez, Prinz podría rebatir diciendo que el acto imaginativo que lleva a la reacción muscular que tiñe la fenomenología conductual con tintes anticipatorios sería inconsciente. Sin embargo, apelar a la idea de una imaginación que es inconsciente es simplemente implausible y trivializa tanto el concepto que este pierde todo su valor. Finalmente, esta potencial réplica parece complejizar de tal manera la visión de Prinz, que torna al concepto de affordance en la alternativa más parsimoniosa para explicar la fluidez y efectividad de nuestras acciones en el mundo.

Uno de los desafíos fundamentales dentro del enfoque fenomenológico de las affordances tiene que ver con definir su presencia en la fenomenología perceptual de un sujeto. Por ejemplo, para Gibson (1966), las affordances son binarias, esto significa que están o no presentes en la fenomenología perceptual de un sujeto. Por ejemplo, un muro puede presentarse como escalable o no. Sin embargo, para McGrenere y Ho (2000) esta visión no logra explicar situaciones que envuelven posibilidades de acciones que podrían ser realizadas con un gran nivel de esfuerzo y dificultad: "for example, a stair that is climbable but only with great difficulty" (p. 2). Estos casos "grises", según los autores, harían pensar que la presencia fenomenológica de las affordances en el campo perceptual de un sujeto sería algo más complejo. Ni Gibson ni McGrene y Ho discuten este asunto más profundamente, por lo tanto, esto se constituye como uno de los desafíos que queda abierto en el proceso de comprender la estructura de las affordances. Sin duda, acá el concepto de solicit de Drayfus y Kelly (2007) podría informar este debate de forma tal que, dependiendo del contexto y la 
acción requerida en este, la variación de solicits y affordances establecería una jerarquía de acción de mayor a menor adaptabilidad contextual. Por una parte, la mayor presión a adoptar una affordance por sobre otra, estaría dada por la presión que ejerce una solicit en el organismo, y esta última se vería regulada por un análisis contextual con mayor alcance que el de los meros objetos colindantes. Si bien este punto es exploratorio, la clarificación de la relación entre contexto, tarea, affordances y solicits parece ser el lugar indicado donde buscar una respuesta para esta discusión.

Ahora bien, desde un punto de vista neurobiológico, el concepto de affordance no tiene un rol descriptivo, sino más bien explicativo. La noción ha sido utilizada para explicar la relación evolutiva entre organismo y ambiente al permitir establecer congruencias y patrones respecto a acciones posibles de un organismo en una situación determinada en relación con sus capacidades sensoriomotoras (Brincker, 2014). Dentro de este enfoque, el concepto de affordance es utilizado como un elemento fundamental para explicar la conformación de nuestra capacidad de planificación y predicción de los beneficios o costos de potenciales acciones, y por lo tanto, su rol explicativo es determinante para entender la forma en que los sujetos se adaptan a su ambiente no solo modificando su conducta, sino su ambiente en lo que se ha denominado construcción de un "nicho adaptativo" (Montesano, Lopes, Bernardino \& Santos-Victor, 2008). De esta forma, la mayoría de los estudios en affordances en el campo de la biología refieren a las posibilidades sensoriomotoras de acción de un organismo y a la forma en que estas nutrirían diversas dimensiones de la percepción humana y su rol adaptativo (Cisek, 2007; Mecklinger, Gruenewald, Weiskopf \& Doeller 2004; Seifert et al. 2014).

Es importante señalar que existen teorías que profundizan otros usos y explicaciones de lo que es una affordance dentro del contexto neurobiológico. Históricamente, las teorías cognitivas han apoyado la idea de la cognición entendida como un sistema representacional de inputs y outputs que permiten estructurar nuestra conducta en base a una cadena de sentidos-pensamientos-acciones (Cisek \& Pezzulo, 2016). Existe evidencia neurofisiológica que sugiere que este modelo explicativo no es aplicable a todos los mecanismos neurológicos que rigen nuestra percepción y acción. Cisek (2007) propone que nuestra cognición se estructura en base a un sistema de control basado en retroalimentaciones directas entre ambiente y organismo, sin mediación de procesamiento representacional, idea consistente con el marco de la psicología ecológica. Este sistema se basaría en estímulos interoceptivos, exteroceptivos y propioceptivos que articulan un sistema sensorial multimodal que permite la resolución de problemas con base en la elección situada y el control adaptativo no mediado por representaciones. Por lo tanto, el objetivo de este sistema no es entender el mundo, sino guiar la interacción con él de forma eficiente en términos adaptativos (Cisek \& Pezzulo, 2016). La teoría de competencia de affordances de Cisek (2007) propone que estas son un conjunto de acciones en el ambiente potencialmente deseables que se presentan en el cerebro como una competencia de posibilidades en base a la información que reciben del ambiente para corroborar su viabilidad demostrando cierta parcialidad respecto a unas por sobre otras debido a su deseo. Así, esta teoría explicaría cómo ciertos animales pueden cambiar rápidamente de un curso de acción a otro debido a que el procesamiento de posibilidades alternativas continúa incluso mientras se está realizando una acción específica. Como la teoría de Cisek (2007) sugiere, el enfoque neurobiológico tiene aún amplio espacio para el desarrollo del concepto de affordances como eslabón estructural de modelos explicativos para la cognición y sus funciones específicas.

Uno de los desafíos más fundamentales que enfrenta el enfoque neurobiológico implica el proveer evidencia estructural y funcional para la existencia 
de las affordances, porque toda la evidencia experimental disponible actualmente es conductual y, por lo tanto, da por hecho, o al menos descansa en, la idea de que el concepto de affordance es la mejor explicación para darle sentido a ciertas conductas observadas (ver por ejemplo, Horton et al. 2012; Kornell, Son \& Terrace, 2007). Pues bien, ¿qué estructuras, procesos cerebrales están a la base de las affordances? Un buen candidato para explorar potenciales respuestas a esta pregunta puede estar en la comprensión del rol de la dopamina en la asignación de valor predictivo a los objetos de la percepción, mediante el análisis de la disfunción dopaminérgica mesoestriatal en condiciones como la psicosis (Howes \& Nour, 2016). La investigación empírica ha relacionado el rol de la dopamina en la asignación de valencia a los objetos de un ambiente. La asignación de esta valencia está guiada principalmente por elementos adaptativos que pueden atraer o repeler la atención de un sujeto en el contexto de tareas adaptativas específicas (Aronson, Wilson, \& Akert, 2017; Roiser, Howes, Chaddock, \& Joyce, 2013). A su vez, la asignación de tal valencia proyecta potenciales beneficios y desventajas en el ambiente, elemento que podría guiar la acción de los individuos mediante un proceso de ponderación adaptativa. Ahora bien, la asignación aberrante de saliencia observada en la psicosis ha sido relacionada experimentalmente con alteraciones dopaminérgicas (Kapur, 2003). Estas alteraciones podrían estar en la base de los cambios fenomenológicos que experimentan los pacientes psicóticos en estados previos a la adopción de delirios, las cuáles, a su vez, han sido interpretadas como cambios en las affordances del ambiente (Fuchs, 2005). Diversos autores han indicado que durante el periodo prepsicótico se reportan diversos cambios en las affordances que perciben los pacientes en el ambiente y en su propio cuerpo (Conrad, 1958; Fuchs, 2005; Kapur, 2003). Esto hace plausible pensar que la acción de la dopamina y sus respectivas alteraciones podrían iluminar la pregunta respecto de los fundamentos neurobiológicos del concepto de affordances. Obviamente, esto constituye una mera hipótesis que necesita mucha más investigación, y por lo mismo, este asunto se constituye como otro desafío que queda abierto en el proceso de clarificar el concepto de affordance. Finalmente, si bien la rivalidad entre los dos enfoques sobre su rol explicativo ha caracterizado la literatura, no es controversial pensar que ambos podrían establecer un programa de investigación complementario. Por una parte, el enfoque fenomenológico podría describir con precisión un fenómeno que parece caracterizar nuestra percepción del mundo; por otra parte, el enfoque neurobiológico podría explorar las bases neuromorfofuncionales que propician la aparición de las affordances en nuestra percepción del mundo. Si bien este enfoque parece mucho más prometedor, no es algo que puedo desarrollar en la presente contribución.

\section{Conclusión}

El concepto de affordance ciertamente captura aspectos fundamentales de la forma en que la mente humana se relaciona y se constituye en su interacción con el medio ambiente. Sin embargo, es claro que el concepto no es unívoco. Este trabajo ha intentado no solamente mapear las características fundamentales del término, sino también formular y analizar algunos de los debates más fundamentales que rodean su comprensión y los desafíos que tales discrepancias dejan abiertos para la continuación de este programa de investigación. La respuesta a estos desafíos exigirá un esfuerzo multidisciplinario. Por ejemplo, la clarificación de la relación entre las diferentes taxonomías de affordances implica no solo análisis filosófico-conceptual, sino práctico. Acá, el desafío es lograr identificar cuál definición del concepto es más operacionalizable. A su vez, ese análisis implicará una retroalimentación del concepto formando una espiral metodológica entre fenómeno y experimentación 
digna de los programas de las ciencias cognitivas actuales. Ahora bien, la exploración del asunto relacionado con la presencia de las affordances en la percepción implicará necesariamente profundizar el concepto de conciencia que ocupa el programa de investigación y revisar la forma en que ciertos elementos preconscientes podrían informar la constitución de los elementos fenoménicos de la experiencia perceptual. Este desafío necesitará ser informado por el trabajo de filósofos de la mente y psicólogos trabajando experimentalmente en la estructura fenoménica de la conciencia humana, en el estilo de la neurofenomenología. Finalmente, la exploración de los fundamentos neurobiológicos del concepto invita a la cooperación entre biólogos, psiquiatras y psicólogos al no solo necesitar del uso de medios experimentales, sino nutrir la discusión a partir de la observación de situaciones psicopatológicas características de la esquizofrenia y otras psicosis. Es de esperar que la exploración del concepto no siga abriendo más debates, sino que también posibilite establecer puentes entre las diversas disciplinas que intentan explorar la naturaleza de la mente y su dependencia con el ambiente.

\section{Referencias}

Aronson, E., Wilson, T., \& Akert, R. (2017). Social psychology. New York: Pearson.

Bayne, T. (2008). The phenomenology of mental agency. Philosophy Compass 3/1, 182-202. Doi: 10.1111/j.1747-9991.2007.00122.x

Bayne, T. (2011). The sense of agency. En F. Macpherson (Ed.), The senses (pp. 355-374). Oxford: Oxford University Press.

Brincker, M. (2014). Navigating beyond "here \& now" affordances on sensorimotor maturation and "false belief" performance. Frontiers in psychology, 5, 1433.

Cisek, P. (2007). Cortical mechanisms of action selection: The affordance competition hypothesis. Philosophical Transactions of the Royal Society of London B: Biological Sciences, 362(1485), 1585-1599.

Cisek, P., \& Kalaska, J. F. (2010). Neural Mechanisms for Interacting with a World Full of Action Choices. Annual Review of Neuroscience, (33), 269-98.

Cisek, P., \& Pezzulo, G. (2016). Navigating the affordance landscape: Feedback control as a process model of behavior and cognition. Trends in Cognitive Sciences, 20(6), 414-424.

Conrad K. (1958). Die beginnende Schizophrekenie. Versuch einer Gestaltanalyse des Wahns. Stuttgart: Thieme.

De Haan, S., \& De Bruin, L. (2010). Reconstructing the minimal self, or how to make sense of agency and ownership. Phenomenology and the Cognitive Sciences, 9, 373-396.

Dreyfus, H. (2002). Intelligence without representation Merleau-Ponty's critique of mental representation the relevance of phenomenology to scientific explanation. Phenomenology and the Cognitive Sciences, 1(4), 367-383.

Dreyfus, H., \& Kelly, S. D. (2007). Heterophenomenology: Heavy-handed sleight-of-hand. Phenomenology and the Cognitive Sciences, 1, 413-425.

Fuchs, T. (2005). Delusional mood and delusional perception. A phenomenological analysis. Psychopathology, 38, 133-139.

Gaver, W. W. (1991). Technology affordances. CHI'91 Conference Proceedings, 79-84.

Gendler, T. (2008). Alief in action (and reaction). Mind \& Language, 23, 552-585.

Gibson, J. J. (1966). The senses considered as perceptual systems. Boston: Houghton-Mifflin.

Gibson, J. (1979). The ecological approach to visual perception. New York: Psychology Press.

Gibson, E. J. (1982). The concept of affordances in development: The renascence of functionalism. En W. A. Collins (ed.), The concept of development. The Minnesota Symposia on Child Psychology (pp. 55-82). Hillsdale, NJ: Lawrence Erlbaum Associates. 
Heft, H. (2001). Ecological psychology in context: James Gibson, Roger Barker, and the legacy of William James's radical empiricism. Mahwah, NJ: Erlbaum.

Heras-Escribano, M., \& Pinedo-García, D. (2018). Affordances and landscapes: Overcoming the nature-culture dichotomy through niche construction theory. Frontiers in psychology, 8, 2294.

Horton. T., Chakraborty, A., \& Amant, R. (2012). Affordances for robots: A brief survey. Avant, 3. 70-84.

Howes O. D., \& Nour M. (2016). Dopamine and the aberrant salience hypothesis of schizophrenia. World Psychiatry, 15(1), 3-4.

Inglod, T. (2011). Being alive: Essays on movement, knowledge and description. Abingdon, UK: Routledge.

Kapur S. (2003). Psychosis as a state of aberrant salience: A framework linking biology, phenomenology, and pharmacology in schizophrenia. American Journal of Psychiatry, 160, 13-23.

Jenkins, H. (2008). Gibson's “affordances": Evolution of a pivotal concept. Journal of Scientific Psychology, 34-45.

Kornell. N, Son, L., \& Terrace, H. (2007). Transfer of metacognitive skills and hint seeking in monkeys. Psychological Science, 18(1), 64-71.

Krueger, J. (2011). Doing things with music. Phenomenology and Cognitive Science, (10), 1-22.

Kusters W. (2014). Filosofie van de waanzin. Lemniscaat: Holland.

McClelland, T. (2015). Affording introspection: An alternative model of inner awareness. Phil Studies. Online first. Doi: 10.1007/s11098-014-0421-x

McClelland, T. (2017a). AI and affordances for mental action. Proceedings of the AISB Annual Convention 2017, 372-379.

McClelland, T. (2017b). The mental affordance hypothesis. Minds Online. Recuperado de http://mindsonline.philosophyofbrains. com/2017/2017-session-1/the-mental-affordance-hypothesis/
McGrenere, J., \& Ho, W. (2000). Affordances: Clarifying and evolving the concept. Proceedings of Graphics Interface. Recuperado de http://teaching.polishedsolid.com/spring2006/iti/read/affordances.pdf

Mecklinger, A., Gruenewald, C., Weiskopf, N., \& Doeller, C. F. (2004). Motor affordance and its role for visual working memory: Evidence from fMrI studies. Experimental Psychology, 51(4), 258-269.

Michaels, C. F. (2003). Affordances: Four points of debate. Ecological Psychology, 15(2), 135-148.

Montesano, L., Lopes, M., Bernardino, A., \& Santos-Victor, J. (2008). Learning object affordances: From sensory-motor coordination to imitation. IEEE Transactions on Robotics, 24(1), 15-26.

Norman, D. A. (1999). Affordance, conventions and designs. Interactions, 38-43.

Payne, E. (2013). Speaking to my madness. USA: CreateSpace.

Prinz, J. (2012). The Conscious Mind. Oxford: OUP. Proust, J. (2016). The evolution of primate communication and metacommunication. Mind \& Language, 31(2), 177-203.

Ratcliffe, M. (2013). Delusional atmosphere and the sense of unreality. In Stanghellini, G., \& Fuchs, T. (Eds.), One Century of Karl Jaspers 'General Psychopathology (pp. 229-244.). Oxford: Oxford University Press.

Rietveld, E., \& Kiverstein. J (2014). A rich landscape of affordances. Ecological Psychology, 26(4), 325-352. Doi: 10.1080/10407413.2014.958035

Roiser, P., Howes, O., Chaddock, C., \& Joyce, M. (2013). Neural and behavioral correlates of aberrant salience in individuals at risk for psychosis. Schizophrenia Bulletin, 39(6), 1328-1336.

Scarantino, A. (2003). Affordances explained. Philosophy of Science, 70, 949-961.

Seifert, L., Wattebled, L., Herault, R., Poizat, G., Adé, D., Gal-Petitfaux, N., \& Davids, K. (2014). Neurobiological degeneracy and affordance perception support functional intra-individual 
variability of inter-limb coordination during ice climbing. Plos One, 9(2), e89865.

Siegel, S. (2010). The contents of visual experience. New York: Oxford University Press.

Siegel, S. (2014). Affordances and the contents of perception. En B. Brogaard (Ed.), Does perception have content? (pp. 39-76). Oxford University Press.

Turvey, M. T. (1992). Affordances and prospective control: An outline of the ontology. Ecol. Psychol. 4, 173-187. Doi: 10.1207/s15326969eco0403_3
Vera, A. H., \& Simon, H. A. (1993). Situated action: A symbolic interpretation. Cognitive Science, 17, 7-48. Doi: $10.1207 / \mathrm{s} 15516709 \operatorname{cog} 1701 \_2$ Walsh, D. M. (2015). Organisms, agency, and evolution. Cambridge University Press. Doi: 10.1017/ CBO9781316402719

Withagen, R., De Poel, H., Araújo, D., \& Pepping, G. (2012). Affordances can invite behavior: Reconsidering the relationship between affordances and agency. New Ideas in Psychology, $30,250-258$. 